\title{
Glued Langmuir-Blodgett Bilayers From Porous Versus
}

\section{Nonporous Surfactants}

Donald H. Mccullough, Vaclav Janout, Junwei Li, James T. Hsu, Quoc Truong, Eugene Wilusz and

Steven L. Regen *

Department of Chemistry and Chemical Engineering, Lehigh University, Bethlehem, Pennsylvania 18015, and the U.S. Army Natick Soldier Center, Natick, Massachusetts 017605

\section{SUPPORTING INFORMATION}

1,2,4,5-tetrakis[N,N-dimethyl-N-(1-hexadecyl)-ammoniummethyl]benzene (2a). A mixture of $90 \mathrm{mg}(0.2 \mathrm{mmol})$ of 1,2,4,5-tetrakisbromomethybenzene, $1.0 \mathrm{~mL}$ of $n$-propanol and $215.6 \mathrm{mg}(0.8 \mathrm{mmol})$ of $\mathrm{N}, \mathrm{N}$-dimethylaminohexadecane was stirred at $80^{\circ} \mathrm{C}$ for $8 \mathrm{~h}$. Removal of solvent under reduced pressure, followed by recrystallization (three times) using $50 \mathrm{~mL}$ acetone/methanol $(80 / 1, \mathrm{v} / \mathrm{v})$ afforded 199 $\mathrm{mg}(65 \%)$ of the title compound as a white solid having $\mathrm{R}_{\mathrm{f}} 0.66$ [silica gel, $\mathrm{CHCl}_{3} / \mathrm{MeOH} / \mathrm{H}_{2} \mathrm{O}(75 / 25 / 2$, $\mathrm{v} / \mathrm{v} / \mathrm{v})$ and ${ }^{1} \mathrm{H} \mathrm{NMR}\left(\mathrm{CD}_{3} \mathrm{OD}\right)$ 8: 8.29(s, $\left.2 \mathrm{H}\right), 5.08(\mathrm{~s}, 8 \mathrm{H}), 3.77$ (bs, $\left.8 \mathrm{H}\right), 3.16(\mathrm{~s}, 24 \mathrm{~h}), 1.88(\mathrm{bs}, 8 \mathrm{H})$, 1.25(m, $104 \mathrm{H}), 0.86(\mathrm{bs}, 12 \mathrm{H}) .{ }^{13} \mathrm{C}-\mathrm{NMR}\left(\mathrm{CDCl}_{3} / \mathrm{CD}_{3} \mathrm{OD}, 10 / 1\right)$ ): 142.42, 132.41, 65.62, 62.60, 31.63, 29.40, 29.26, 29.07, 26.02, 22.70, 22.38, 13.79, 13.66; mp 226-227 (decomp). Anal. Calcd for $\mathrm{C}_{82} \mathrm{H}_{166} \mathrm{~N}_{4} \mathrm{Br}_{4} .2 \mathrm{H}_{2} \mathrm{O}: \mathrm{C}, 62.97 ; \mathrm{H}, 10.90 ; \mathrm{N}, 3.58$. Found: C, 62.92; H, 10.59; N, 3.60.

1,2,4,5-Tetrakis-[N,N-dimethyl-N-(1-dodecyl)-ammoniummethyl]benzene (2b). A mixture of 90 $\mathrm{mg}(0.2 \mathrm{mmol})$ of 1,2,4,5-tetrakisbromomethybenzene, $1.0 \mathrm{~mL}$ of $n$-propanol and $170.8 \mathrm{mg}(0.8 \mathrm{mmol})$ of $\mathrm{N}, \mathrm{N}$-dimethylaminododecane was stirred at $80^{\circ} \mathrm{C}$ for $5 \mathrm{~h}$. Removal of solvent under 
reduced pressure, followed by recrystallization (three times) using $40 \mathrm{~mL}$ of acetone/methanol $(40 / 1, \mathrm{v} / \mathrm{v})$ afforded $181 \mathrm{mg}(69 \%)$ of the title compound as a white solid having $\mathrm{R}_{\mathrm{f}}$ 0.59, [silica gel, $\mathrm{CHCl}_{3} / \mathrm{MeOH}_{2} \mathrm{H}_{2} \mathrm{O}(75 / 25 / 2, \mathrm{v} / \mathrm{v} / \mathrm{v})$ and ${ }^{1} \mathrm{H} \mathrm{NMR}\left(\mathrm{CDCl}_{3}\right)$ 8: 8.29(s, $\left.2 \mathrm{H}\right), 5.32(\mathrm{~s}, 8 \mathrm{H}), 4.08$ (bs, $\left.8 \mathrm{H}\right)$, 3.40(s, $24 \mathrm{H}), 1.84(\mathrm{bs}, 8 \mathrm{H}), 1.28(\mathrm{~m}, 72 \mathrm{H}), 0.88(\mathrm{t}, 12 \mathrm{H}) .{ }^{13} \mathrm{C}-\mathrm{NMR}\left(\mathrm{CDCl}_{3} / \mathrm{CD}_{3} \mathrm{OD}, 10 / 1\right) \delta: \quad 142.47$, 132.36, 65.59, 62.57, 31.56, 29.27, 29.15, 29.00, 25.99, 22.65, 22.32, 13.71,13.59; mp 227-229 (decomp). Anal. Calcd for $\mathrm{C}_{66} \mathrm{H}_{134} \mathrm{~N}_{4} \mathrm{Br}_{4} .2 \mathrm{H}_{2} \mathrm{O}$ : C, 59.97; H, 10.36, N, 4.23. Found: C, 59.69; H, 9.97; N, 4.26.

Tris(N,N-dimethyl-N-hexadecylammoniummethyl)mesitylene (3). To a hot solution of $0.115 \mathrm{~g}$ $(0.288 \mathrm{mmol})$ of tris-bromomethymesitylene in $1.0 \mathrm{~mL}$ of $n$-propanol was added $0.234 \mathrm{~g}(0.868 \mathrm{mmol})$ of N,N-dimethylaminohexadecane. The reaction mixture was stirred at $80^{\circ} \mathrm{C}$ for $24 \mathrm{~h}$, cooled to rt and diluted with $50 \mathrm{~mL}$ of acetone. Subsequent crystallization at $-10^{\circ} \mathrm{C}$ afforded $0.200 \mathrm{~g}$ of crude product. Repeated recrystallization (three times) from acetone/methanol (50/1,v/v) afforded $120 \mathrm{mg}(35 \%)$ of the title compound having $\mathrm{R}_{\mathrm{f}} 0.48$ [silica gel, $\mathrm{CHCl}_{3} / \mathrm{MeOH} / \mathrm{H}_{2} \mathrm{O}\left(75 / 25 / 2\right.$, v/v/v) and ${ }^{1} \mathrm{H} \mathrm{NMR}\left(\mathrm{CDCl}_{3} / \mathrm{CD}_{3} \mathrm{OD}\right.$, 20/1 [v/v]) 8: 4.98(s, $6 \mathrm{H}), 3.68(\mathrm{~m}, 6 \mathrm{H}), 15(\mathrm{~s}, 18 \mathrm{H})$, 2.64(s, $9 \mathrm{H}), 1.74(\mathrm{~m}, 6 \mathrm{H}), 1.20(\mathrm{~m}, 78 \mathrm{H}), 0.82(\mathrm{t}, 9 \mathrm{H})$.

${ }^{13} \mathrm{C}-\mathrm{NMR}\left(\mathrm{CDCl}_{3} / \mathrm{CD}_{3} \mathrm{OD}, 10 / 1\right) \delta: 147.31,127.55,65.69,63.62,31.64,29.41,29.08,26.09,22.60,22.39$, 22.02, 21.87, 13.80, 13.69; mp 162-164 (decomp). Anal. Calcd for $\mathrm{C}_{66} \mathrm{H}_{132} \mathrm{~N}_{3} \mathrm{Br}_{3} .2 \mathrm{H}_{2} \mathrm{O}: \mathrm{C}, 63.75 ; \mathrm{H}$, 11.02; N, 3.39. Found:C, 63.97; H, 10.67; N, 3.45. 